\title{
Improved Genetic Algorithm based on Search Parameters Dynamically Adjust and its Application
}

\author{
Yongsheng Jia ${ }^{1, a}$, Xiujian Lv ${ }^{2, b}$ \\ ${ }^{1}$ Shijiazhuang Vocational and Technology Institute, Department of Information Engineering \\ Shijiazhuang,China \\ ${ }^{2}$ Shijiazhuang Vocational and Technology Institute, Department of Information Engineering \\ Shijiazhuang,China \\ a studentjys@163.com, 'blvjii@126.com
}

Keywords: genetic algorithms, real coding, premature convergence

\begin{abstract}
Practical application of genetic algorithm is easy to premature convergence and accuracy of search results is not high. Optimal value for the premature convergence and low accuracy to dynamically adjust the search parameters to optimize the calculation, the whole process of evolution, the algorithm always maintain a strong global search ability and local search capabilities; test results show that genetic such improved algorithm is effective, easy to fall into local optimum, and can greatly improve the accuracy of the optimal solution..
\end{abstract}

\section{Introduction}

Beginning in the 1980s, countries around the world have developed a number of IT security evaluation criteria. Within these standards, Trusted Computer System Evaluation Criteria (TCSEC), known as Orange Book, released by the U.S. Department of Defense is the earliest one. Other standards are basically the basis of it [1].

Initial TCSEC is for isolated computer systems, especially for minicomputers and mainframe systems, and this standard applies only to military and government, not for the enterprise. TCSEC and ITSEC are not involved in open systems, and they are static model, only reflecting the static security situation [2]. CTCPEC has a certain development based on them but failed to break above limitations. FC makes additions and modifications on the TCSEC, defined in protection profiles and security targets, cleared detailed outline of the system security requirements provided by the user, but it has not been formally put into use because of some defects. CC defines the basis guidelines as the assessment of IT products and systems security. Compared with the early evaluation criteria, its advantage is the openness of its structure, completeness of expression mode and usefulness [3].

\section{Algorithm introduction}

The steps of the improved genetic algorithm are:

1) Initialization, input for solving problem of all kinds of data and control parameters: the population scale crossover probability and mutation probability algorithm termination scale

Accurate, initial standard deviation D, standard deviation D maximum, minimum, Compressibility factor C (used to adjust evolution process of standard deviation).

2) Using decimal floating point number coding, random generation meet the constraint condition of initial groups. Find out each individual adaptive value.

3) Using the survival of the fittest, natural selection rules of the survival of the fittest, according to individual performance tournament selection.

4) According to the crossover probability solution set use the best performance of the individual.

$X(1)$ and choose the individual $X(\mathrm{~m})$ cross operation: $g=X(n)+r^{*}(X(1)-X(n)), \mathrm{r}$ is $[0,1]$ between the random number; If g satisfy the constraint conditions, then use g replace $X(\mathrm{n})$, storage 
corresponding adaptive value, or continue to cross operation; After the crossover operation, looking for the current adaptive value of the best individual instead of $X(1)$.

5) If the standard deviation $D$ in the evolutionary process is greater than the maximum or less than the minimum, change the compressibility factor $\mathrm{C}$, make standard deviation continued to shrink and continuous expanding continuously alternating between, standard deviation narrowed, can improve the accuracy of search, so that the optimal value of accuracy is improved; Standard deviation change, make the search scope change, in the larger range to search for, and jump out of local optimal and avoid premature convergence.

6) Based on mutation probability of population individuals to gauss mutation, the current performance of the best individual does not participate in variation, individual variation to its legitimacy after inspection, or with boundary value instead of the value after the variation, or a new variation; After the variation, with the current adaptive value optimal individual instead of $X(1)$.

7) Change variance $\mathrm{D}=\mathrm{D} * \mathrm{C}$, including $\mathrm{C}$ for the coefficient factor.

8) If not satisfied to suspend the condition turn the third step; Otherwise end circulation, the optimal solution to the current problems as the optimal solution.

\section{Algorithm performance analysis}

In order to validate the efficiency of the method and reliability, we take the following tests.

Example 1: (results from literature [2], literature [3] also described.

$$
f(x)=-2 \pi x-\sum_{i=1}^{11} \sin \left(2^{i} \pi x\right)
$$

$x \in[0,1]$, solve min $f(x)$.

Table 1. Algorithm contrast

\begin{tabular}{|c|c|c|c|}
\hline & The optimal solution & The optimal value & Cpu consumption \\
\hline $\begin{array}{c}\text { Algorithm in } \\
\text { this paper }\end{array}$ & 0.566713 & -8.817893 & \multirow{2}{*}{$0.1875 \mathrm{~s}$} \\
\cline { 2 - 3 } & 43037885 & 51158466 & \\
\hline Algorithm 1 $^{[2]}$ & 0.5667138 & -8.817891 & $0.39 \mathrm{~s}$ \\
\hline Algorithm 2 $^{[2]}$ & 0.5667129 & -8.817884 & $0.27 \mathrm{~s}$ \\
\hline
\end{tabular}

Example $2^{[4]}$ uses genetic algorithm solving real root of Heonardo equation:

$f(x)=x^{3}+2 x^{2}+10 x-20=0$.

Table 2. Algorithm results in this paper

\begin{tabular}{|c|c|c|c|c|}
\hline Content & Root. & Error & $\begin{array}{c}\text { The number of call } \\
\text { objective function }\end{array}$ & $\begin{array}{c}\text { Cpu } \\
\text { consumption }\end{array}$ \\
\hline \multirow{2}{*}{$\begin{array}{c}\text { This paper } \\
\text { algorithm }\end{array}$} & 1.368808 & 7.105427 & 2400 & $0.1875 \mathrm{~s}$ \\
\cline { 2 - 3 } & 10782137 & $357601002 \times 10^{-15}$ & 2400 & 0.100 \\
\hline
\end{tabular}

The calculated results show that the algorithm is of high precision.

Example 3 [5]:

$$
\begin{aligned}
& f(x, y, z)=\sqrt{x}+\sqrt{y}+\sqrt{z}, \\
& \text { s.t. } x^{2}+2 y^{2}+3 z^{2} \leq 1, \\
& x \geq 0, y \geq 0, z \geq 0,
\end{aligned}
$$

Solve $\max f(x, y, z)$.

Table 3. Algorithm results in this paper

\begin{tabular}{|c|c|c|c|c|}
\hline $\begin{array}{c}\text { Algorithm } \\
\text { content.... }\end{array}$ & Optimal solution & Optimal value & Cpu consumption & $\begin{array}{c}\text { Calling objective } \\
\text { function }\end{array}$ \\
\hline \multirow{4}{*}{\begin{tabular}{c} 
Algorithm of this $\begin{array}{c}\text { Alg } \\
\text { paper }\end{array}$ \\
\cline { 2 - 3 }
\end{tabular}} & $\mathrm{x}=0.633249$ & & \\
\cline { 2 - 3 } & $\mathrm{y}=0.39038476$ & \multirow{2}{*}{$0.4375 \mathrm{~s}$} & 5800 \\
\cline { 2 - 3 } & 62443492 & 47828498 & \\
\hline $\mathrm{z}=0.306031$ & & \\
\hline
\end{tabular}




\begin{tabular}{|l|c|c|c|c|}
\hline & 13709523 & & & \\
\hline & $\mathrm{x}=0.636$ & \multirow{2}{*}{1.980062} & & \\
\cline { 1 - 1 } \cline { 4 - 5 } & $\mathrm{y}=0.395$ & 14308170 & - & - \\
\hline & $\mathrm{z}=0.307$ & & & \\
\hline
\end{tabular}

Example 4 [6]: The following is given in southern Shenyang HunHe coast four drainage outlet sewage treatment efficiency nonlinear programming problem:

$$
\begin{aligned}
\min F= & 696.744 x_{1}^{1962}+10586.71 x_{1}^{59898}+63.927 \times x_{2}^{18815}+9054.54 x_{2}^{59898}+375.658 x_{3}^{29972}+ \\
& 520091 x_{3}^{59898}+113.471 x_{4}^{18815}+223.825 x_{4}^{5}+23.626 \times x_{4}^{48344}+5431427 x_{4}^{459898}+3982
\end{aligned}
$$

s.t.

$20.475\left(1-x_{1}\right) \leq 22.194$

$17.037\left(1-x_{1}\right)+12.998\left(1-x_{2}\right) \leq 23.505$

$15.660\left(1-x_{1}\right)+11.942\left(1-x_{2}\right)+8.822\left(1-x_{3}\right) \leq 24.031$

$14.229\left(1-x_{1}\right)+10.855\left(1-x_{2}\right)+8.026\left(1-x_{3}\right)+21.965\left(1-x_{4}\right) \leq 24.576$

$x_{i} \in[0,0.9]$

$i=1,2,3,4$

The $x_{i}$ is the sewage treatment efficiency of $\mathrm{i}$-th discharge outlet. $\mathrm{F}$ is cost function, unchanged object function, directly using the proposed algorithm to get global optimal solution:

$x_{1}=0.48803412536133$,

$x_{2}=0.50580461032083$,

$x_{3}=0.50575593047643$,

$x_{4}=0.63760784396065$.

The minimum cost is 5060. 952102127397 (ten thousand yuan).

The optimal solution of literature [5] is:

$x_{1}=0.488358$,

$x_{2}=0.505813$,

$x_{3}=0.506156$,

$x_{4}=0.637249$.

The minimum cost is 5 060. 959742354245 (ten thousand yuan).

\section{Conclusions}

This paper puts forward a kind of improved genetic algorithm, through the use of change parameters and change the range of searching, can make the population in the evolution from premature convergence effectively, population constantly to optimal value approximation, and ultimately to search the optimal value. Verify the proposed algorithm is a very robust algorithm, in the population scale is lesser also can search to the optimal value. Computing time and the relationship between the population scale is larger, the population more hours spent more time. Make sure genetic algorithm for computing time and parameters of the relationship or a problem need to be researched.

\section{References}

[1] M. R. Azimi-Sadjadi, R. J. Lion. Fast Learning Process of Multilayer Neural Networks Using Resursive Least Squares Method. IEEE Trans.On SP, Vol.40(2010), p.446 450.

[2] Kennedy, J. and Eberhart. R. C. Particle swarm optimization. In Proc. IEEE Iternational Conference on Neural Networks, IEEE Service Center, pisataway, NJ, 2010, p. 39 43.

[3] Duta S, Shekhar S. Bond rating: a non-conservative application of neural networks. Proc IEEE Int Conf on Neural Networks[C].San Diego: IEEE, 2011, p. 443 450. 
[4] Sobajic DJ, Pao YH. Artificial neural net based dynamic security assessment for electric power systems.IEEE Trans.On Power Systems, Vol.4(2009), p.220 226.

[5] Gordon R.T. Artificial neural network approach to assessment. Intelligent Engineering Systems Through Networks, Vol.4(2007), p.1175 1180.

[6] Molina AM Chou K C. Application of Neural Networks for the Performance Evaluation of Bridges. Probabilistic Mechanics and Structural and Geotechnical Reliability, Proceedings of the Specialty Conference. Sponsored by ASCE,2008, p.298 301.

[7] Ge Hong, Mao Zong-yuan. The Analysis of t he Local Search Efficiency of Genetic Neural Networks and t he Improvement of Algorithm [A]. Processing of $t$ he $4 t$ h World Congress on Intelligent Cont rol and Automation[ C] . Hefei : Press of East China University of Science and Technolgy ,2002.

[8] Fogel D B. Asymptotic Convergence Properties of Genetic Algorithms and Evolutionary Programming : Analysis and Experiments[J] .Cybernetics and System ,1994,25 (6) :389 - 407.

[9] Gambardella L M , Dorigo M. Solving symmetric and asymmetric TSPs by ant colonies[A]. In Proceedings of t he IEEE international conference on evolutionary computation (ICEC '96) [C]. USA : IEEE Press , 1996. 622 - 627.

[10]Yanmei Li, Jingmin Wang. Risk Assessment Model of Smart Grid Project Based on Variable P recision RS and LSSVM. Advances in Information Sciences and Service Sciences (AISS). Vol.3, N o.10, 2011,11:375 383. 